\title{
SALICILATOS ISOLADOS DE FOLHAS E TALOS DE Salix martiana LEYB. (SALICACEAE)
}

Carromberth Carioca Fernandes

Centro de Ciências Biológicas e da Natureza, Universidade Federal do Acre, 69990-000 Rio Branco - AC, Brasil

Lorena Mayara de Carvalho Cursino, Jussival de Abreu Pinheiro Novaes, Camilla Avelino Demetrio, Orlando Libório Pereira Júnior e Cecília Verônica Nunez*

Coordenação de Pesquisas em Produtos Naturais, Instituto de Pesquisa da Amazônia, Av. André Araújo, 2936, 69060-001 Manaus - AM, Brasil

Iêda Leão do Amaral

Coordenação de Pesquisas em Botânica, Instituto de Pesquisa da Amazônia, Av. André Araújo, 2936, 69060-001 Manaus - AM, Brasil

Recebido em 24/8/08; aceito em 19/11/08; publicado na web em 2/4/09

\begin{abstract}
SALICYLATES ISOLATED FROM LEAVES AND STEMS OF Salix martiana Leyb. (SALICACEAE). Salix martiana Leyb. is an endemic species from the Amazon river floodplain areas (varzeas), of the State of Amazonas. Stems and leaves were extracted with dichloromethane, methanol and hydro-alcohol and these extracts were fractionated by using conventional chromatographic techniques. The major substances isolated, salicin and trichocarposide (6-0-p-coumaroyl salicin), were determined through analyses of NMR 1D $\left({ }^{1} \mathrm{H}\right.$ and $\left.{ }^{13} \mathrm{C}\right)$ and NMR 2D (gHSQC and gHMBC). These compounds were isolated for the first time in Salix martiana Leyb. (Salicaceae). The percentage of these compounds in $S$. martiana is very high. The extracts were analyzed for their DPPH antioxidant capacity and the methanolic from the leaves and the hydro-alcoholic from the stems were the more active.
\end{abstract}

Keywords: Salix martiana; salicin; trichocarposide.

\section{INTRODUÇÃO}

A família Salicaceae tem uma distribuição espacial em diversos biomas do mundo, com 43 gêneros e aproximadamente 1000 espécies. ${ }^{1}$ No Brasil, ocorrem 19 gêneros e cerca de 80 espécies. ${ }^{1}$ A família é conhecida pela presença de salicilatos, que são glicosídeos fenólicos. Estudos químicos prévios de espécies de Salicaceae forneceram principalmente as seguintes substâncias: salicina, salicortina, $2^{\prime}$-cinamoilsalicortina, salicina di-glicosilada, salicilol, 2 '-O-acetilsalicina, 2'-O-acetilsalicortina e $m$-hidroxibenzil- $\beta$ - $D$ glicosídeo; diversas atividades farmacológicas foram descritas para estas substâncias, destacando-se a analgésica, ${ }^{2}$ antiinflamatória ${ }^{3} \mathrm{e}$ antioxidante. $^{4}$

A espécie Salix martiana Leyb., escolhida para o presente estudo, é sinonímia de Salix humboldtiana var. martiana (Leyb.) Anders. A espécie é de ampla distribuição na região de várzeas do Rio Solimões, Amazonas. Popularmente é conhecida como oeirana ou oeirana branca e utilizada para diabetes. A falta de relatos de estudos químicos prévios para a espécie motivou o presente trabalho fitoquímico. $\mathrm{Na}$ literatura disponível, este é o primeiro relato dos dados de RMN de ${ }^{13} \mathrm{C}$ para a substância trichocarposídeo ou 6-0-p-coumaroil-salicina.

\section{PARTE EXPERIMENTAL}

\section{Procedimentos experimentais gerais}

Os espectros de $\mathrm{RMN}$ de ${ }^{1} \mathrm{He}{ }^{13} \mathrm{C}$ e experimentos bidimensionais foram realizados em espectrômetro Varian Inova-500 ${ }^{\circledR}$ operando a $500 \mathrm{MHz}$ para o núcleo de ${ }^{1} \mathrm{H}$ e $125 \mathrm{MHz}$ para o núcleo de ${ }^{13} \mathrm{C}$, TMS foi utilizado como referência interna. Os deslocamentos químicos foram dados em ppm, tendo como referência interna o tetrametilsilano

*e-mail: cecilia@inpa.gov.br
TMS ( $\delta=0,0 \mathrm{ppm}$ ) ou o próprio solvente. Os solventes utilizados foram $\mathrm{CDCl}_{3}, \mathrm{CD}_{3} \mathrm{OD}$ e $\mathrm{D}_{2} \mathrm{O}$.

Os espectros de massas foram obtidos em espectrômetro de massas tipo ion trap, modelo Esquire HCT (Bruker Daltonics), acoplado a um cromatógrafo líquido modelo Prominence (Shimadzu), composto por três bombas modelo LC-20AD, injetor automático SIL-20AC, forno para coluna CTO-20AC, controladora CBM-20A, detector DAD (diode array) SPD-M20A e três fontes de ionização: electrospray, APCI e nanospray. A fonte de ESI usada nas análises foi operada a $3500 \mathrm{~V}$ (tanto ESI ${ }^{+}$como ESI'), com um fluxo de nitrogênio de 15 psi (nebulizer) e $5 \mu \mathrm{L} /$ min (drying gas) a $250{ }^{\circ} \mathrm{C}$. O trap foi operado com hélio em seu interior, em uma diferença de pressão de $4 \times 10^{-6}$ bar em relação ao basal (sem hélio) e o tempo de abertura do trap foi de $50 \mathrm{~ms}$. O ICC (ion charge control) estava em 70000 para ESI ${ }^{+}$e 30000 para ESI- Para as cromatografias em coluna (CC) utilizou-se sílica gel 60 (0,063-0,200 mm), Amberlite XAD-4 e alumina neutra, todos da Merck, como fase estacionária. Para as cromatografias em camada delgada comparativa (CCDC) e em camada delgada preparativa (CCDP) empregou-se sílica gel 60 G e 60 GF254 (Merck), respectivamente. A visualização dos compostos em CCDC foi realizada por irradiação com luz ultravioleta em 254 e $366 \mathrm{~nm}$, vapores de iodo e/ou por pulverização com solução de $\mathrm{Ce}\left(\mathrm{SO}_{4}\right)_{2}$ seguida de aquecimento.

\section{Material vegetal}

As folhas e talos das folhas de Salix martiana Leyb. (Salicaceae) foram coletados às margens do lago do Catalão, região inundada pelo Rio Solimões (área de várzea), próximo de Manaus, em 22/7/2004. A exsicata encontra-se devidamente depositada, sob o número 224265 , no Herbário do Instituto Nacional de Pesquisa da Amazônia - INPA, em Manaus - AM. 


\section{Extração e isolamento}

As folhas (200 g) e os talos das folhas (200 g) foram secos em temperatura ambiente, moídos e extraídos consecutivamente com os solventes diclorometano, metanol e metanol/ $\mathrm{H}_{2} \mathrm{O}$ 7:3, utilizando aparelho de ultrassom por $20 \mathrm{~min}$, cada procedimento repetido 3 vezes, obtendo-se os extratos ST.DCM (2,6 g), ST.MeOH (9,0 g), e ST.MA (5,5 g) (para os talos) e SF.DCM (5,4 g), SF.MeOH (8,8 g), e SF.MA (11,3 g) (para as folhas).

Cada extrato foi submetido a vários processos de cromatografia em coluna (CC) à pressão atmosférica, com o intuito de obter frações separadas em solventes de polaridade crescente, para posterior fracionamento. As sub-frações que continham os compostos majoritários foram submetidas a vários processos cromatográficos em coluna (CC) e camada delgada preparativa (CCDP) até obtenção das substâncias salicina (1) e trichocarposídeo ou 6-0-p-coumaroil-salicina (2), Figura 1. Os rendimentos das substâncias estão mostrados na Tabela 1 .

Tabela 1. Rendimento de obtenção das substâncias isoladas nos diferentes extratos de Salix martiana

\begin{tabular}{lccc}
\hline Parte vegetal (g) & Extrato (g) & $\begin{array}{c}\text { Substância } \\
\text { isolada }\end{array}$ & $\begin{array}{c}\text { Rendimento } \\
(\%)\end{array}$ \\
\hline & SF.DCM & & \\
& $(5,4)$ & & \\
Folhas (200) & SF.MeOH & Salicina & 1,75 \\
& $(8,8)$ & Trichocarposídeo & 3 \\
& SF.MA & & \\
& $(11,3)$ & & 1,93 \\
Talos $(200)$ & ST.DCM & Salicina \\
& $(2,6)$ & \\
& ST.MeOH & \\
& $(9,0)$ & \\
& ST.MA & \\
& $(5,5)$ & \\
\end{tabular}

\section{Avaliação qualitativa da atividade antioxidante usando DPPH}

A avaliação da atividade antioxidante foi realizada qualitativamente por meio do ensaio com 2,2-difenil-1-(2,4,6-trinitrofenil) hidrazila (DPPH), através da borrifação da placa cromatográfica, previamente eluída e seca, com uma solução 0,2\% de DPPH em metanol (MeOH). O tempo de revelação varia de 5 a 30 min, sendo que a placa adquire cor púrpura e a presença de substâncias antioxidantes é revelada onde há coloração branco-amarela. ${ }^{5}$

\section{Avaliação quantitativa da atividade antioxidante usando DPPH}

A atividade antioxidante foi avaliada através do método de redução da capacidade de seqüestro (CS) de radicais com 2,2-difenil1-(2,4,6-trinitrofenil)hidrazila (DPPH). Os resultados obtidos foram expressos em equivalência com o ácido ascórbico, termo este adotado para expressar a razão da concentração da amostra pela concentração equivalente de ácido ascórbico. A concentração equivalente de ácido ascórbico foi obtida pela interpolação dos dados de variação de absorbância e concentração de ácido ascórbico na curva dose-resposta.

Portanto estes dois termos são distintos e o significado do termo "equivalência com o ácido ascórbico" permite correlacionar a resposta obtida do extrato vegetal com a do antioxidante padrão e quanto mais próximo de 1,0 mais semelhante é a capacidade antioxidante da amostra à do ácido ascórbico, escolhido como antioxidante padrão por ser ativo nos organismos vivos.

\section{Preparação da curva dose-resposta do ácido ascórbico para o} ensaio com DPPH

Pesaram-se 0,0100 g de ácido ascórbico e em seguida diluiu-se em 25 mL de metanol grau PA, resultando na solução de concentração de $400 \mu \mathrm{g} / \mathrm{mL}$. A solução de DPPH ${ }^{\bullet}$ foi preparada dissolvendo-se $0,0030 \mathrm{~g}$ do reagente em $100 \mathrm{~mL}$ de $\mathrm{MeOH}$ grau PA.

O procedimento consistiu na adição de $1000 \mu \mathrm{L}$ do radical $\mathrm{DPPH}{ }^{\bullet}$ em microtubos Eppendorf e em seguida adicionou-se 99 ( $1^{\circ}$ tubo); 97 ( $2^{\circ}$ tubo); 96 ( $3^{\circ}$ tubo); 95 ( $4^{\circ}$ tubo); 90 (5॰ tubo); 86 ( $6^{\circ}$ tubo) e 82 ( $7^{\circ}$ tubo) $\mu \mathrm{L}$ de $\mathrm{MeOH}$ grau PA e completou-se com $1 \mu \mathrm{L}$ de ácido ascórbico ( $1^{\circ}$ tubo); 3 ( $2^{\circ}$ tubo); 4 ( $3^{\circ}$ tubo); 5 ( $4^{\circ}$ tubo); 10 ( $5^{\circ}$ tubo); 14 ( $6^{\circ}$ tubo) e 18 ( $7^{\circ}$ tubo). O preparo do branco consistiu em $1000 \mu \mathrm{L}$ do radical $\mathrm{DPPH}^{\bullet}$ com $100 \mu \mathrm{L}$ de $\mathrm{MeOH}$ grau PA. A absorbância foi medida em $517 \mathrm{~nm}$ após 20 min de reação.

\section{Ensaio de redução do DPPH}

A solução de $\mathrm{DPPH}^{\bullet}$ foi preparada dissolvendo-se $0,0030 \mathrm{~g}$ do reagente em $100 \mathrm{~mL}$ de $\mathrm{MeOH}$ grau PA.

A reação do teste amostra foi iniciada pela adição de $1000 \mu \mathrm{L}$ desta solução, $12 \mu \mathrm{L}$ de $\mathrm{MeOH}$ grau PA e $88 \mu \mathrm{L}$ de amostra (0,5 mg/ $\mathrm{mL}$ ). O preparo do branco consistiu em $1012 \mu \mathrm{L}$ de $\mathrm{MeOH}$ grau PA com $88 \mu \mathrm{L}$ da amostra, enquanto o preparo do controle consistiu em $1100 \mu \mathrm{L}$ da solução de $\mathrm{DPPH}^{\bullet}$. A variação de absorbância ( $\triangle \mathrm{ABS}$ ) foi obtida pela seguinte equação:

$\Delta \mathrm{ABS}=\mathrm{ABS}$ controle $-(\mathrm{ABS}$ amostra $-\mathrm{ABS}$ branco $)$

onde: $\mathrm{ABS}$ controle = absorbância obtida do controle; $\mathrm{ABS}$ amostra = absorbância obtida da amostra; ABS branco = absorbância obtida do branco.

As leituras foram realizadas após 20 min de reação em $517 \mathrm{~nm}$.

\section{RESULTADOS E DISCUSSÃO}

A substância 1, isolada dos extratos metanólico das folhas e diclorometânico dos talos de $S$. martiana, foi identificada através das análises dos espectros de $\mathrm{RMN}$ de ${ }^{1} \mathrm{H}$ e de ${ }^{13} \mathrm{C}$, mono e bidimensionais ( $g$ HSQC e $g$ HMBC) e comparada com dados da literatura. ${ }^{6}$ Os principais sinais observados são na região dos hidrogênios aromáticos e hidrogênios carbinólicos de açúcares.

Na região de hidrogênios aromáticos, observam-se os sinais centrados em $\delta 7,33(d d, 1 \mathrm{H}, J=7,5$ e 1,5 Hz, H-3) que acopla com $\delta 7,03(d d d, 1 \mathrm{H}, J=7,5 ; 7,5 \mathrm{e} 1,0 \mathrm{~Hz}, \mathrm{H}-4)$ com constante orto $(7,5$ $\mathrm{Hz})$ e $\operatorname{com} \delta 7,26(d d d, 1 \mathrm{H}, J=8,0 ; 7,5$ e $1,5 \mathrm{~Hz}, \mathrm{H}-5)$ com constante meta $(1,5 \mathrm{~Hz})$. O sinal em $\delta 7,03$, além de acoplar com $\delta 7,33$, acopla $\operatorname{com} \delta 7,26 \operatorname{com} J=7,5 \mathrm{~Hz}$, posicionando em posição orto. E, finalmente, em $\delta 7,21(d d, 1 \mathrm{H}, J=8,0$ e 1,0 Hz, H-6) acopla com $J=8,0$ (orto) $\operatorname{com} \delta 7,26$ e com $\delta 7,03 \operatorname{com} J=1,0$ (meta). Em $\delta 4,79(d, J$ $=13,0 \mathrm{~Hz}, \mathrm{H}-7 \mathrm{a})$ e em $\delta 4,59(d, J=13,0 \mathrm{~Hz}, \mathrm{H}-7 \mathrm{~b})$ atribuídos aos hidrogênios benzílicos. Observou-se finalmente, no anel glicosídico, um dubleto centrado em $\delta 4,87(d, J=8,0 \mathrm{~Hz}, \mathrm{H}-1$ ') anomérico, cujo acoplamento de $8,0 \mathrm{~Hz}$ é característico da configuração $\beta$; os demais sinais confirmam tratar-se da $\beta$-glicose. A análise dos espectros de RMN bidimensionais ( $g$ HSQC e $g$ HMBC) forneceu as informações relativas às conectividades entre os carbonos e os hidrogênios e estão mostradas na Tabela 2 e Figura 1.

Através dos deslocamentos químicos observados em interações heteronucleares direta ${ }^{13} \mathrm{C}-{ }^{1} \mathrm{H}$ gHSQC, foi possível determinar a atribuição de cada carbono e de seu respectivo hidrogênio, sendo: 7,33 (H-3) e 128,71 (C-3); 7,26 (H-5) e 128,79 (C-5); 7,21 (H-6) e 115,93 (C-6); 7,03 (H-4) e 122,56 (C-4); [4,78 e 4,57 (H-7a e b)] e 
59,82 (C-7); 4,87 (H-1') e 102,24 (C-1'); 3,51 (H-2') e 73,92 (C-2'); 3,49 (H-3') e 76,85 (C-3'); 3,41 (H-4') e 70,22 (C-4'); 3,42 (H-5') e 77,09 (C-5'); [3,91 e 3,71 (H-6')] e 61,39 (C-6').

Através dos deslocamentos químicos observados em interações heteronucleares a longa distância ${ }^{13} \mathrm{C}-{ }^{1} \mathrm{H} g \mathrm{HMBC}$ (Figura 1) foi possível observar a atribuição de cada correlação, sendo: C-1 acopla

Tabela 2. $\delta_{\mathrm{H}}$ e $\delta_{\mathrm{C}}$ de $\mathbf{1}$ e 2 , em $\mathrm{CD}_{3} \mathrm{OD}, 500 \mathrm{MHz}$ para ${ }^{1} \mathrm{H}$ e 125 $\mathrm{MHz}$ para ${ }^{13} \mathrm{C}$

\begin{tabular}{|c|c|c|c|c|}
\hline \multirow[t]{2}{*}{ Posição } & \multicolumn{2}{|l|}{1} & \multicolumn{2}{|l|}{2} \\
\hline & ${ }^{1} \mathrm{H}$ (multipli-cidade) & ${ }^{13} \mathrm{C}$ & ${ }^{1} \mathrm{H}$ (multi-plicidade) & ${ }^{13} \mathrm{C}$ \\
\hline 1 & quaternário & 156,0 & quaternário & 157,0 \\
\hline 2 & quaternário & 131,0 & quaternário & 132,4 \\
\hline 3 & $\begin{array}{c}7,33(1 \mathrm{H}, d d, J=7,5 \\
1,5 \mathrm{~Hz})\end{array}$ & 128,7 & $\begin{array}{c}7,32(1 \mathrm{H}, d, J=7,0 \\
\mathrm{Hz})\end{array}$ & 129,9 \\
\hline 4 & $\begin{array}{c}7,03(1 \mathrm{H}, d d d, \\
J=7,5 ; 7,5 ; 1,0 \mathrm{~Hz})\end{array}$ & 122,6 & $\begin{array}{c}6,98(1 \mathrm{H}, d d d, \\
J=7,0 ; 6,5 ; 2,0 \mathrm{~Hz})\end{array}$ & 123,9 \\
\hline 5 & $\begin{array}{c}\text { 7,26 }(1 \mathrm{H}, d d d, \\
J=8,0 ; 7,5 ; 1,5 \mathrm{~Hz})\end{array}$ & 128,8 & $\begin{array}{c}7,15(1 \mathrm{H}, d d, J=6,5 ; \\
1,5 \mathrm{~Hz})\end{array}$ & 129,9 \\
\hline 6 & $\begin{array}{c}7,21(1 \mathrm{H}, d d, J=8,0 \\
1,0 \mathrm{~Hz})\end{array}$ & 115,9 & $\begin{array}{c}7,13(1 \mathrm{H}, d d, J=8,0 ; \\
1,5 \mathrm{~Hz})\end{array}$ & 117,3 \\
\hline 7 & $\begin{array}{c}4,78(1 \mathrm{H}, d, J=13,0 \\
\mathrm{Hz}) \text { e } 4,57(1 \mathrm{H}, d \\
J=13,0 \mathrm{~Hz})\end{array}$ & 59,8 & $\begin{array}{c}4,78(1 \mathrm{H}, d, J=13,0 \\
\mathrm{Hz}) \text { e } 4,57(1 \mathrm{H}, d, \\
J=13,0 \mathrm{~Hz})\end{array}$ & 61,0 \\
\hline 1 ' & $\begin{array}{c}4,87(1 \mathrm{H}, d, J=8,0 \\
\mathrm{Hz})\end{array}$ & 102,2 & $\begin{array}{c}4,88(1 \mathrm{H}, d, J=7,5 \\
\mathrm{Hz})\end{array}$ & 103,3 \\
\hline 2 ' & $\begin{array}{c}3,46(1 \mathrm{H}, t, J=9,0 ; \\
8,0 \mathrm{~Hz})\end{array}$ & 76,9 & $\begin{array}{c}3,53(1 \mathrm{H}, d, J=7,5 \\
\mathrm{Hz})\end{array}$ & 75,1 \\
\hline $3^{\prime}$ & $3,42(m)$ & 77,1 & $\begin{array}{c}3,49(1 \mathrm{H}, t, J=9,0 \\
\mathrm{Hz})\end{array}$ & 78,0 \\
\hline $4^{\prime}$ & $\begin{array}{c}3,41(1 \mathrm{H}, d d, J=9,0 \\
7,5 \mathrm{~Hz})\end{array}$ & 70,2 & $\begin{array}{c}3,44(1 \mathrm{H}, t, 1 \mathrm{H} \\
\quad J=9,0 \mathrm{~Hz})\end{array}$ & 71,8 \\
\hline 5 , & $\begin{array}{c}3,51(1 \mathrm{H}, d d, J=7,5 \\
7,5 \mathrm{~Hz})\end{array}$ & 73,9 & $3,70(m)$ & 75,7 \\
\hline 6 & $\begin{array}{c}3,90(1 \mathrm{H}, d d \\
J=12,0 ; 2,0 \mathrm{~Hz}) \mathrm{e} \\
3,71(1 \mathrm{H}, d d, 12,0 \\
5,0 \mathrm{~Hz})\end{array}$ & 61,4 & $\begin{array}{c}4,54(1 \mathrm{H}, d d, \\
J=12,0 ; 2,5 \mathrm{~Hz}) \\
\text { e } 4,37(1 \mathrm{H}, d d, \\
J=12,0 ; 7,0 \mathrm{~Hz})\end{array}$ & 64,6 \\
\hline $1 "$ & & & quaternário & 161,4 \\
\hline $2 "$ & & & $\begin{array}{c}6,82(2 \mathrm{H}, d, J=8,5 \\
\mathrm{Hz})\end{array}$ & 116,9 \\
\hline $3 "$ & & & $\begin{array}{c}7,46(2 \mathrm{H}, d, J=8,5 \\
\mathrm{Hz})\end{array}$ & 131,3 \\
\hline $4 "$ & & & quaternário & 127,2 \\
\hline $5 "$ & & & $\begin{array}{c}7,46(2 \mathrm{H}, d, J=8,5 \\
\mathrm{Hz})\end{array}$ & 131,3 \\
\hline $6 "$ & & & $\begin{array}{c}6,82(2 \mathrm{H}, d, J=8,5 \\
\mathrm{Hz})\end{array}$ & 116,9 \\
\hline $7 "$ & & & $\begin{array}{c}7,62(1 \mathrm{H}, d, J=16,0 \\
\mathrm{Hz})\end{array}$ & 146,9 \\
\hline $8 "$ & & & $\begin{array}{c}6,34(1 \mathrm{H}, d, J=16,0 \\
\mathrm{Hz})\end{array}$ & 115,0 \\
\hline $9 "$ & & & quaternário & 169,0 \\
\hline
\end{tabular}

com H-3, H-5, H-6, H-7a e b e H-1'; C-2 acopla com H-4, H-6; C-6 acopla com $\mathrm{H}-4$, todos em $J_{3}$. Apenas o C-2 acopla com H-7a e b em $J_{2}$. No anel glicosídico, observa-se: C-1' acopla com H-2' e C-5' acopla com $\mathrm{H}-4$ ', ambos em $J_{2}$.

Do extrato metanólico das folhas foi possível isolar também a substância 2, cujos sinais observados nos espectros de RMN ${ }^{1} \mathrm{H}$ e de ${ }^{13} \mathrm{C}$, comparados parcialmente com "salicina" (Tabela 2), mostram uma grande similaridade entre os deslocamentos químicos dos carbonos e hidrogênios, sendo da porção "salicina", porém, observam-se mais 9 carbonos, todos $\mathrm{sp}^{2}$. A análise dos espectros de RMN bidi-
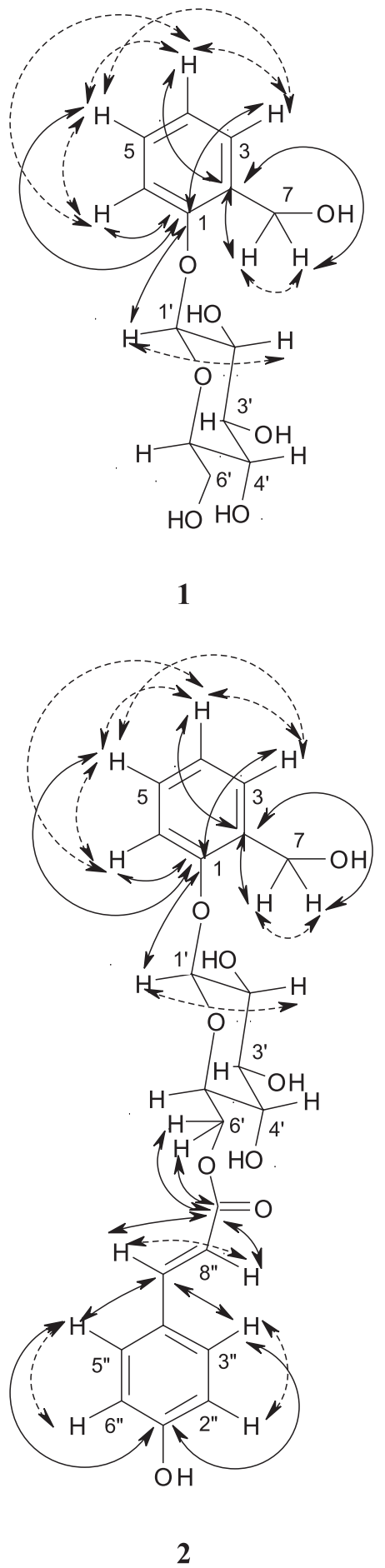

Figura 1. Acoplamentos observados nos espectros de RMN de gCOSY (セ- $\rightarrow)$ e de $g H M B C(\leftrightarrow)$ para 1 e 2 
mensionais ( $g \mathrm{HSQC}$ e $g \mathrm{HMBC}$ ) forneceu as informações relativas às conectividades entre os carbonos e os hidrogênios e estão mostradas na Tabela 2 e Figura 1.

No outro anel aromático, observam-se dois dupletos centrados em $\delta 6,82(d, 2 \mathrm{H}, J=8,5 \mathrm{~Hz})$ e $\delta 7,46(d, 2 \mathrm{H}, J=8,5 \mathrm{~Hz})$ que acoplam um com o outro, atribuídos aos hidrogênios H-2"/H-6" e H-3"/H-5". Dois dupletos centrados em $\delta 7,62(d, 1 \mathrm{H}, J=16,0 \mathrm{~Hz})$ e $\delta 6,34(d, 1 \mathrm{H}$, $J=16,0 \mathrm{~Hz}$ ), atribuídos aos hidrogênios H-7" e H-8", respectivamente (em acoplamento trans). Os acoplamentos observados no $g \mathrm{HMBC}$ entre o $\delta$ em 7,62 - H7" e o $\delta 168,99$ - C9" e entre este e os dois H em $\delta 4,54$ e 4,37 permitiram a completa identificação estrutural.

Ainda permanecia a dúvida se os anéis estariam ligados através do $\mathrm{O}$ entre 7 e 1". A análise por espectrometria de massas tipo ion trap, utilizando ESI tanto o modo positivo quanto negativo, possibilitou a conclusão de que a massa da molécula era de $432 \mathrm{~m} / \mathrm{z}$, a qual confere com a proposta $\mathbf{2}$.

As substâncias salicina (1) majoritária e trichocarposídeo ou 6-0-p-coumaroil-salicina (2) foram isolados pela primeira vez na espécie Salix martiana Leyb. (Salicaceae), abundante nas áreas de várzeas do Rio Solimões, Amazonas. Os altos rendimentos destas duas substâncias na planta estudada podem vir a torná-la nova fonte de matéria-prima para obtenção do ácido acetil-salicílico, através

Tabela 3. Avaliação da atividade antioxidante dos extratos das folhas e dos talos de Salix martiana em equivalente em ácido ascórbico

\begin{tabular}{|c|c|c|c|c|c|c|}
\hline \multirow{3}{*}{$\begin{array}{c}\text { Parte } \\
\text { Vegetal/ } \\
\text { Extrato }\end{array}$} & \multicolumn{6}{|c|}{ Método usando DPPH• } \\
\hline & \multicolumn{3}{|c|}{ Valores médios } & \multicolumn{3}{|c|}{ Desvio-padrão } \\
\hline & $\begin{array}{c}\mid \Delta \mathrm{ABS} \\
517 \mid\end{array}$ & $\begin{array}{c}{[\mathrm{AA}]} \\
\mathrm{eq}\end{array}$ & Equiv. & $\begin{array}{c}\mid \Delta \mathrm{ABS} \\
517 \mid\end{array}$ & $\begin{array}{c}{[\mathrm{AA}]} \\
\mathrm{eq}\end{array}$ & Equiv. \\
\hline \multicolumn{7}{|l|}{ Folhas } \\
\hline DCM & 0,030 & 0,412 & 12,149 & 0,002 & 0,017 & 0,491 \\
\hline $\mathrm{MeOH}$ & 0,050 & 0,570 & 8,772 & 0,002 & 0,012 & 0,186 \\
\hline MA & 0,004 & 0,209 & 24,049 & 0,003 & 0,020 & 2,322 \\
\hline \multicolumn{7}{|l|}{ Talos } \\
\hline DCM & 0,045 & 0,383 & 12,798 & 0,002 & 0,013 & 0,440 \\
\hline $\mathrm{MeOH}$ & 0,015 & 0,293 & 17,175 & 0,004 & 0,028 & 1,604 \\
\hline MA & 0,051 & 0,581 & 8,657 & 0,007 & 0,051 & 0,769 \\
\hline
\end{tabular}

de processos industriais de semi-síntese. Estudos sobre a toxicidade desses extratos estão sendo realizados.

A avaliação da atividade antioxidante mostra que os extratos mais promissores são os extratos metanólico das folhas e metanol/água dos talos (Tabela 3). Os demais são inativos. Uma vez que o extrato metanólico das folhas foi rico em salicina e trichocarposídeo, e há ainda a presença de sinais nos espectros de RMN de outros derivados da salicina, em fase de purificação, é possível que sejam os compostos responsáveis pela atividade. A avaliação em CCDC, borrifada com solução de DPPH, das substâncias salicina e trichocarposídeo indicou uma moderada atividade antioxidante. No entanto, a avaliação quantitativa ainda não foi realizada.

\section{AGRADECIMENTOS}

À Fundação de Amparo à Pesquisa do Estado do Amazonas (FAPEAM) e ao Programa de Pesquisas em Biodiversidade, componente Temático, (PPBio/INPA/MCT) pelos auxílios financeiros. C. C. Fernandes agradece à Superintendência da Zona Franca de Manaus (SUFRAMA), pela Bolsa de Doutorado de Demanda Social concedida. L. M. C. Cursino agradece à FAPEAM pela bolsa de Iniciação Científica concedida. Ao Prof. Dr. M. Yoshida, L. C. Roque e R. C. Pereira Jr. do Centro de Biotecnologia da Amazônia (CBA), pela realização dos espectros de RMN. Ao Profs. Drs. E. Pinto, N. P. Lopes e o doutorando F. Dorr pelas análises de espectrometria de massas.

\section{REFERÊNCIAS}

1. Souza, V. C.; Lorenzi, H.; Botânica Sistemática: guia ilustrado para identificação das famílias de Angiospermas da flora brasileira, baseado em APGII, Instituto Plantarum: Nova Odessa, 2005.

2. Yunes, R. A.; Cechinel Filho, V.; Ferreira, J.; Calixto, J. B.; Stud. Nat. Prod. Chem. 2005, 30, 191.

3. Smith, I. D.; Temple, D. M.; Shearman, R. P.; Prostaglandins 1975, $10,41$.

4. Zhang, X. F.; Thuong, P. T.; Min, B.-S.; Ngoc, T. M.; Hung, T. M.; Lee, I. S.; Na, M. K.; Seong, Y.-H.; Song, K.-S.; Bae, K. H.; J. Nat. Prod. 2006, 69, 1370.

5. Cuendet, M.; Hostettmann, K.; Potterat, O.; Dyatmiko, W.; Helvetica Chim. Acta 1997, 80, 1145.

6. Zapesochnaya, G. G.; Kurkin, V. A.; Braslavskii, V. B.; Filatova, N. V.; Chem. Nat. Compd. 2002, 38, 314. 